\title{
Computer Modeling of Hippocampal CA1 Pyramidal Cells - a Tool for in Silico Experiments
}

\author{
Metz Júlia, Szilágyi T*, Perian M, Orbán-Kis K \\ Department of Physiology, University of Medicine and Pharmacy of Tîrgu Mures, Romania
}

\begin{abstract}
Objective. In silico experiments use mathematical models that capture as much as possible from the properties of the biological system under investigation. Our aim was to test the publicly available CA1 pyramidal cell models using the same simulation tasks, to compare them, and provide a systematic overview of their properties in order to improve the usefulness of these models as a tool for in silico experiments.

Methods. Parameters describing the morphology of the cells and the implemented biophysical mechanisms were collected from the ModelDB database of SenseLab Project. This data was analyzed in correlation with the purpose for which each particular model was developed. Multicompartmental simulations were run using the Neuron modeling platform. The properties of the action potentials generated in response to current injection, the firing pattern and the dendritic back-propagation were analyzed.

Results. The studied models were optimized to explore different physiological and pathological properties of the CA1 pyramidal cells. We could identify four broad classes of models focusing on: (i) initiation of the action potential, firing pattern and spike timing, (ii) dendritic backpropagation, (iii) dendritic integration of synaptic inputs and (iv) neuronal network activity. Despite the large variation of the active conductances implemented in the models, the properties of the individual action potentials were quite similar, but even the most complex models could not reproduce all studied biological phenomena.
\end{abstract}

Conclusions. At the moment the "perfect" pyramidal cell model is not yet available. Our work, hopefully, will help finding the best model for each scientific question under investigation.

Keywords: computational neuroscience, hippocampus, pyramidal cell, shared database

Received: 17 November 2014 / Accepted: 15 December 2014

\section{Introduction}

With the extremely fast development of the information technology in the last decades, a new research method became available for broad masses of neuroscientists. In silico experiments use mathematical models that capture as much as possible from the properties of the biological system under investigation. Realistic computer models are built using biological data, and the results of simulations are then compared with results of biological experiments [1]. In this way inconsistencies in the model can be identified, and in an iterative way the model can be refined and improved.

Until very recently, the computing power and informatics tools required to build biologically realistic models were available only in specialized facilities. Today even desktop computers can run relatively complex neuronal simulations, and the models can be validated against detailed data from biological experiments. Modern neuronal simulation environments hide the mathematical details from the user, therefore the researchers can focus their attention to the biological processes [2]. In this way the in silico experiments can spread from the high performance computational neuroscience centers to the ordinary experimental laboratories.

Sharing of computational neuroscience models is beneficial for the whole scientific community because the complexity of the models makes difficult to build completely

* Correspondence to: Tibor Szilágyi

E-mail: szilagyi@umftgm.ro new ones [3]. One published implementation can be used as it is for in silico experiments, or as a building block for modified versions by other researchers, or it can be the origin for other models with extended range of applicability.

The hippocampus plays a key role in spatial representation [4] and long term memory consolidation [5, 6]. This brain area is also important because it is affected by several diseases as epilepsy [7, 8], Alzheimer's disease $[9,10]$ or schizophrenia [11]. Axons of the pyramidal cells in area CA1 constitute the main hippocampal output, therefore it is essential to understand the physiological role of these neurons under normal and pathological conditions. It is not surprising, therefore, that the CA1 pyramidal neuron is one of the most studied class of neuron in the brain. Many publicly available models of this cell type exist, but each of these is validated only against a certain biological dataset.

The aim of our work was to test the publicly available CA1 pyramidal cell models using the same simulation tasks, to compare them, and provide a systematic overview of their properties in order to improve the usefulness of these models as a tool for in silico experiments.

\section{Methods}

Our work intends to help using an in silico tool in the experimental laboratories. To be useful in neuroscience labs, the modeling environment have to support morphologically detailed single cells containing active membrane conductances through ion channels, and also networks of 
morphologically detailed cells. It also should run on all popular operating systems and should be distributed free of charge. The simulation environment which satisfies all these conditions, and also has the largest user base is the NEURON simulation platform (http://www.neuron.yale. edu) [12], which as of May 2014, has more than 1450 scientific articles and books reporting work that was done with it [13]. Based on the above considerations our study is restricted to models implemented in this simulation environment. Detailed model descriptions were obtained from ModelDB (https://senselab.med.yale.edu/modeldb/)

Sixty CA1 pyramidal cell models were analyzed. Models without dendritic tree or with very simplified dendritic geometry were not included in our study. Parameters describing the morphology of the cells and the implemented biophysical mechanisms were collected for all models. In many cases we retrieved the used parameter set from the shared script, as it was not described in detail in the published research article.

Regarding the morphology of the cells we recorded the number of apical and basal compartments, whether the axon was explicitly modeled, and the way the dendritic spines were taken into account. For the passive electrical properties the following were recorded: the membrane capacitance, the axial resistance, the specific membrane resistance, the equilibrium potential of the passive conductance, the resting membrane potential and the simulated temperature. The implemented ion channels and their maximum conductance were separately noted for the soma, dendrites and axon. Where applicable, the intracellular calcium handling mechanisms were also taken into account.

The morphological and biophysical parameters and the purpose for which was developed each particular model were analyzed. Multicompartmental simulations were run for the most often used models. The active conductances were implemented by Hodgkin-Huxley type equations. Responses of model cells to current injection were recorded. The properties of the generated action potentials (threshold, amplitude, rate of depolarization, rate of repolarization, duration), the firing pattern (maximum firing frequency, spike frequency adaptation) and the dendritic back-propagation were analyzed.

Throughout our paper each model is referred to by the name of the first author of the research article using the model. If an author had more than one published model, these are distinguished by letters (e.g. Author_a, Author_b, etc.).

\section{Results}

The studied models were optimized to explore different physiological and pathological properties of the CA1 pyramidal cells. We could identify four broad classes of models:

1. signal generation: initiation of the action potential, firing pattern and spike timing,

2. the back-propagation of the action potentials in the dendritic tree,
3. signal integration: dendritic integration of synaptic inputs, its dependence on membrane properties, neuronal morphology and synaptic properties,

4. neuronal network activity: modeling physiological and pathological oscillations and their modulation, and the role of different cell types in shaping the network activity.

1. Models focusing on signal generation (firing properties) explored:

- the relation between the voltage dependence of the sodium current and the properties of the action potential (Royeck, [14]),

- the role of the axonal M-type potassium current in determining the threshold of action potential initiation and the resting membrane potential (Shah_a, [15]),

- the mechanism of action of certain drugs (Ferrante [16]),

- the effect of different mutations of the of the M-type $\mathrm{K}^{+}$-channel protein that decrease the channels voltage sensitivity (Miceli [17]),

- the effects of different inactivation pathways of the A-type $\mathrm{K}^{+}$-channel (Fineberg [18]).

2. Models focusing on the back-propagation of the action potentials in the dendritic tree studied:

- the modulation of the amplitude of the back-propagated action potentials by the properties of the local voltage-dependent ion channels (especially the Atype $\mathrm{K}^{+}$-channel) and external factors, as the timing of the synaptic inputs (Migliore_a [19]),

- the effect of the morphology of the dendritic tree and the shape of the action potential on the efficiency of back-propagation (Golding_a [20]),

- the influence of the local density of the A-type $\mathrm{K}^{+}$ channels and the morphology of the dendritic tree on the back- and forward propagation of the action potential in the oblique apical dendrites (Migliore_d [21]),

3. Models focusing on somato-dendritic signal integration explored:

- the increased attenuation of the EPSPs (excitatory postsynaptic potentials) in the apical dendrites (Golding_b [22]),

- the effect of the perforant path and Schaffer collateral inputs, and the conditions under which the dendritic spikes initiated by perforant path inputs propagate to the cell body (Jarsky [23]),

- the post-inhibitory rebound spiking that can be unmasked by the reduction of the A-type $\mathrm{K}^{+}$-current (Ascoli [24]),

- the effect of dendritic distribution of A-type potassium and h-conductances (hyperpolarization-activated) on the integration of subthreshold perforant path inputs (Migliore_b [25]),

- the effect of $I_{h}$ on the firing probability of a CA1 pyramidal cell receiving synaptic inputs activated with 
different degree of synchronization and situated at different distances from the cell body (Migliore_c [26]),

- the up- and down-regulation of $I_{h}$ by potassium channels dependent on the $I_{h}$ peak conductance (Migliore_e [27]),

- the effect of certain anti-depressive drugs on the dendritic excitability (Poolos [28]),

- the temporal and spatial requirements for the initiation of dendritic spikes (Gasparini [29]),

- the mechanisms that facilitate the propagation of the $\mathrm{Ca}^{2+}$ spikes (Ashhad [30]),

- the relation between the synchronization of the back-propagating action potentials to the EPSPs and the induction of long-term potentiation (Watanabe [31]),

- the frequency and the regularity of firing as a function of the frequency and spatial distribution of the synaptic inputs, neuronal morphology, active membrane conductances, the synchrony and regularity of the synaptic inputs ( $\mathrm{Li}[32])$, the effect of the syn- chrony of the synaptic input on the input-output relation of the neuron ( $\mathrm{Li}[33])$,

- the linear or non-linear synaptic integration that can explain the summation of the synaptic inputs, identification of the dendritic regions, which can operate as an independent functional subunit, and the inputoutput functions of these subunits, which could best represent the integrative properties of the cell (Poirazi $[34,35])$,

- the progressive destruction of the theta resonance and the phase response on distal dendrites of the pyramidal cells during latent and chronic epilepsy, which correlates with the availability of the h-current (Marcelin [36]),

- the effect of different forms of noise on the spatial and temporal integration of synaptic inputs (Graham [37]),

- mapping realistic neurons into equivalent reduced models running faster while maintaining a very high accuracy of the membrane potential dynamics during synaptic inputs (Marasco [38])

Table I. The morphology of the modeled neurons

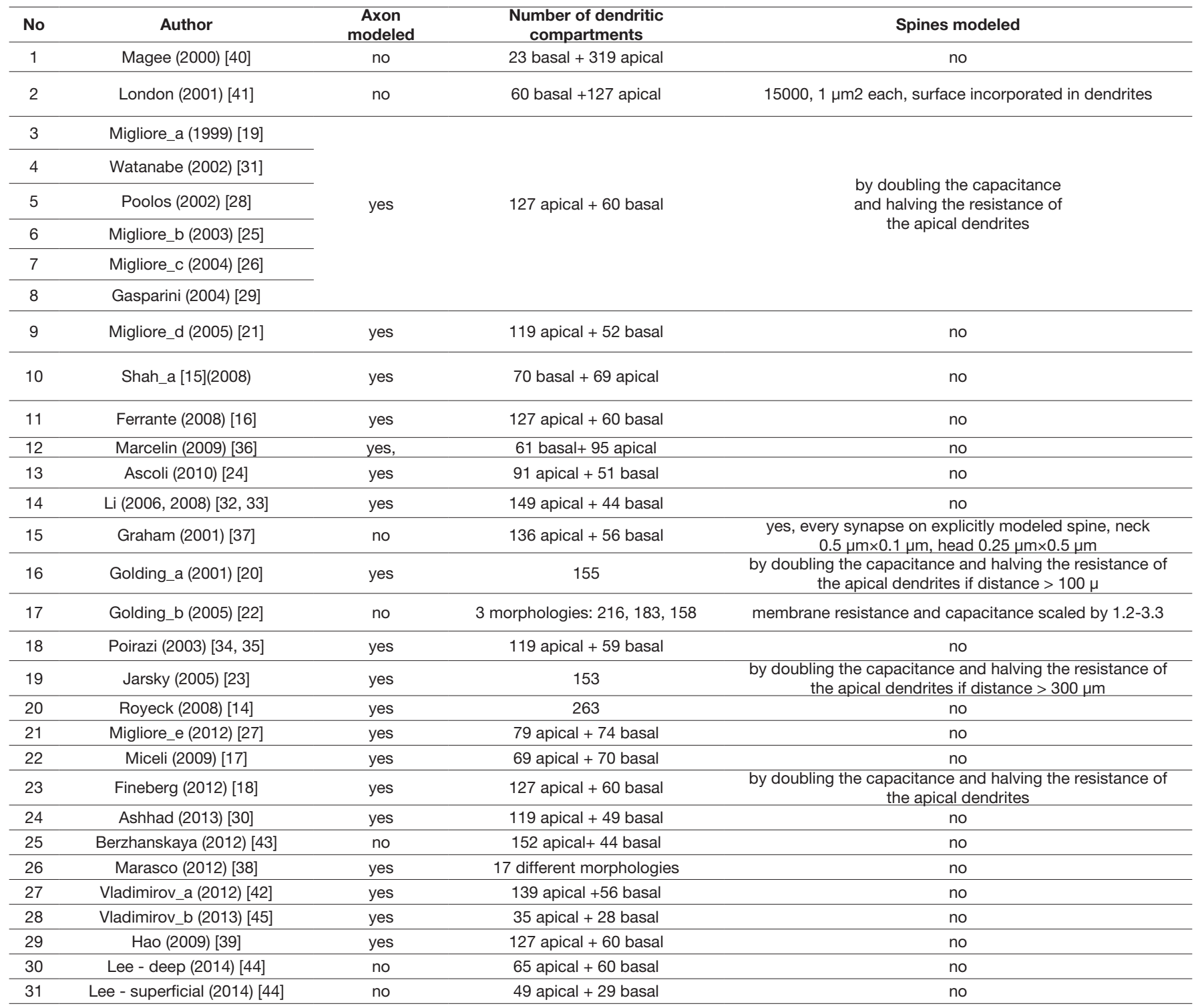


- a simple arithmetic rule for spatial summation of concurrent excitatory glutamatergic inputs and inhibitory GABAergic inputs, defining a nonlinear term reflecting the strength of shunting effect (Hao [39]),

- the factors contributing to the reduced variability of the somatic EPSPs depending on the localization of the synapses on passive dendrites (Magee [40]),

- the effects of synaptic scaling under in vivo conditions in a passive neuronal model (London [41]).

4. Models focusing on network activity also used different models of the CA1 pyramidal cells (Vladimirov_a[42]), (Berzhanskaya [43]), (Lee [44]), (Vladimirov_b [45]). (Table $I-V)$

Table II. The passive properties of the model cells

\begin{tabular}{|c|c|c|c|c|c|c|c|}
\hline No & Author & $\begin{array}{l}\text { Membrane } \\
\text { capacitance } \\
(\mu \mathrm{F} / \mathrm{cm} 2)\end{array}$ & $\begin{array}{l}\text { Axial } \\
\text { resistance } \\
(\Omega \cdot \mathrm{cm})\end{array}$ & $\begin{array}{c}\text { Specific } \\
\text { membrane } \\
\text { resistance }(\Omega \cdot \mathrm{cm} 2)\end{array}$ & $\begin{array}{l}\text { Equilibrium potential } \\
\text { of the passive } \\
\text { conductance }(\mathrm{mV})\end{array}$ & $\begin{array}{c}\text { Resting } \\
\text { membrane } \\
\text { potential (mV) }\end{array}$ & $\begin{array}{l}\text { Simulated } \\
\text { temperature } \\
\left({ }^{\circ} \mathrm{C}\right)\end{array}$ \\
\hline 1 & Magee [40] & 1 & 70 & 10000 & -65 & -65 & 6.3 \\
\hline 2 & London [41] & 1 & $\begin{array}{c}100 \\
70 \\
150\end{array}$ & $\begin{array}{l}40000 \\
10000 \\
40000\end{array}$ & 0 & 0 & 6.3 \\
\hline 3 & Migliore_a [19] & 1 & $150, a: 50$ & 28000 & -65 & -65 & 34 \\
\hline 4 & Watanabe [31] & 1 & 150 , a: 50 & 28000 & -65 & -65 & 34 \\
\hline 5 & Poolos [28] & 1 & $150, a: 50$ & 28000 & -65 & -65 or -70 & 34 \\
\hline 6 & Migliore_b [25] & 1 & $150 \Delta$, a: 50 & $28000 \&$ & -65 & -65 & 34 \\
\hline 7 & Migliore_c [26] & 1 & 150, a: 50 & 28000 & -65 & -65 & 34 \\
\hline 8 & Gasparini [29] & 1 & $150, a: 50$ & 28000 & -65 & -65 & 34 \\
\hline 9 & Migliore_d [21] & 1 & $150, a: 50$ & 28000 & -65 & -65 & 34 \\
\hline 10 & Shah_a [15] & 1 & 150, a: 50 & 28000 & -65 & -65 & 34 \\
\hline 11 & Ferrante [16] & 1 & 150 , a: 50 & 28000 & -65 & -65 & 35 \\
\hline 12 & Marcelin [36] & 1 & 150, a: 50 & 28000 & -70 & -70 & 35 \\
\hline 13 & Ascoli [24] & 3.2 & 50 & 14600 & -62 & -62 & 35 \\
\hline 14 & $\mathrm{Li}[32,33]$ & 1 & 50 & 28000 & -65 & -65 & 35 \\
\hline 15 & Graham [37] & 1 & 200 & 30000 & -65 & -65 & 35 \\
\hline 16 & Golding_a [20] & $\begin{array}{l}0.75 \\
\text { a (myelin): } \\
0.075\end{array}$ & 200 & 40000 & -65 & -65 & 35 \\
\hline 17 & Golding_b [22] & 1.70944 & 139.09 & 40000\# & -65 & -72 & 35 \\
\hline 18 & Poirazi $[34,35]$ & 1 & 50-35\# & 200000-12000\# & to set $\mathrm{RMP}=-70$ & -70 & 34 \\
\hline 19 & Jarsky [23] & $\begin{array}{l}0.75 \\
\text { a (myelin): } \\
0.04\end{array}$ & 200 & 40000 & -66 & -70 & 35 \\
\hline 20 & Royeck [14] & 0.75 & 150 & 70000 & -70 & -70 & 30 \\
\hline 21 & Migliore_e [27] & 1.9 & 80; a: 50 & 20000 & -70 & -70 & 35 \\
\hline 22 & Miceli [17] & 0.75 & 500; a: 50 & 28000 & -65 & -65 & 35 \\
\hline 23 & Fineberg [18] & 1, d: 2 & 150; a: 50 & 28000 , d: 56000 & -65 & -65 & 34 \\
\hline 24 & Ashhad [30] & 1 & $50-35 \$$ & $72000-30000 \$$ & -65 & -65 & 34 \\
\hline 25 & Berzhanskaya [43] & 1 & 80 & 28000 & * & -65 & 35 \\
\hline 26 & Marasco [38] & 1 & 150; a: 50 & 28000 & -65 & -65 & 35 \\
\hline 27 & Vladimirov_a [42] & 0.9 & 200; a 100 & s, d: 50000, a: 1000 & -70 & -65 & 6.5 \\
\hline 28 & Vladimirov_b [45] & 0.75 & 200, a: 100 & s, d: 50000, a: 1000 & -65 & -70 & 6.5 \\
\hline 29 & Hao [39] & 1 & 80 & $6000 \$$ & -70 & -70 & 34 \\
\hline 30 & Lee - deep [44] & 1 & 295 & 24150 & -104 & -104 & 34 \\
\hline 31 & Lee - superficial [44] & 1 & 295 & 32900 & -104 & -104 & 34 \\
\hline \multicolumn{4}{|c|}{$\begin{array}{l}\text { s: soma, d: dendrites, a: axon } \\
\text { RMP: resting membrane potential } \\
\text { the resting membrane potential of every compartment is } 0 \\
\text { \# varies sigmoidally with distance from soma }\end{array}$} & \multicolumn{3}{|c|}{$\begin{array}{l}\text { \$ exponentially decreasing in apical trunk with } \\
\Delta \text { linearly increasing with distance from soma } \\
\text { \& linearly decreasing with distance from soma }\end{array}$} & \\
\hline
\end{tabular}

\section{Models with multiple applications}

Some models were used in several different simulations. We further investigated in detail these models.

The Migliore_d [21] model with slight modifications was used by several authors, being a realistic model with very simple biophysical properties (only a sodium conductance for soma/dendrites and axon, an h-current and two potassium conductances are implemented). This model was used to demonstrate how the oblique apical dendrites of these neurons may bind different inputs to generate an output signal [46]. The possible role of the CA1 pyramidal neurons in the emergence of schizophrenic behavior was also investigated [47]. The model was also used to study 
Table III. Active conductances implemented in the soma of the model cells and their maximum conductance $(\mathrm{mS} / \mathrm{cm} 2)$

\begin{tabular}{|c|c|c|c|c|c|c|c|c|c|c|c|c|c|c|c|}
\hline No & Author & 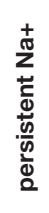 & 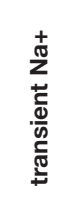 & 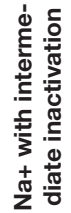 & $\varepsilon$ & 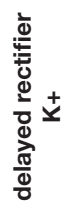 & 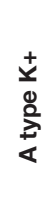 & 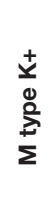 & $\begin{array}{l}\text { 呈 } \\
\text { 足 } \\
\text { 立 }\end{array}$ & 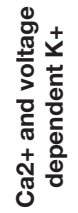 & 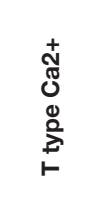 & 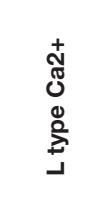 & 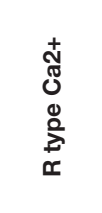 & 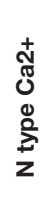 & 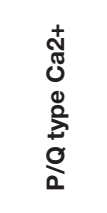 \\
\hline 1 & Magee [40] & - & - & - & - & - & - & - & - & - & - & - & - & - & - \\
\hline 2 & London [41] & - & - & - & - & - & - & - & - & - & - & - & - & - & - \\
\hline 3 & Migliore_a [19] & - & 32 & - & - & 10 & 48 & - & - & - & - & - & - & - & - \\
\hline 4 & Watanabe [31] & - & 32 & - & - & 10 & 48 & - & - & - & - & - & - & - & - \\
\hline 5 & Poolos [28] & - & 32 & - & 0.6 & 3 & 8 & - & - & - & - & - & - & - & - \\
\hline 6 & Migliore_b [25] & - & 32 & - & 0.5 & 3 & 48 & - & - & - & - & - & - & - & - \\
\hline 7 & Migliore_c [26] & - & 30 & - & 0.5 & 1 & 10 & - & - & - & - & - & - & - & - \\
\hline 8 & Gasparini [29] & - & 30 & - & 0.05 & 5 & 22 & - & - & - & - & - & - & - & - \\
\hline 9 & Migliore_d [21] & - & 25 & - & 0.05 & 10 & 10 & - & - & - & - & - & - & - & - \\
\hline 10 & Shah_a $[15]$ & - & 135 & - & 0 & 180 & 40 & 20 & 0.01 & - & 0.1 & - & - & - & - \\
\hline 11 & Ferrante [16] & - & 30 & - & 0.06 & 1 & 10 & - & - & - & - & - & - & - & - \\
\hline 12 & Marcelin [36] & - & - & - & 0.05 & - & - & - & - & - & - & - & - & - & - \\
\hline 13 & Ascoli [24] & - & 24 & - & 0.02 & 8 & 4 & - & - & - & - & - & - & - & - \\
\hline 14 & Li $[32,33]$ & - & 20 & - & 0.05 & 10 & 25 & - & - & - & - & - & - & - & - \\
\hline 15 & Graham [37] & $\begin{array}{l}\text { L } \\
0\end{array}$ & - & - & 0.03 & - & 0.2 & - & - & - & 1 & - & - & - & - \\
\hline 16 & Golding_a [20] & 42 & - & - & - & 40 & 100 & - & - & - & - & - & - & - & - \\
\hline 17 & Golding_b [22] & - & - & - & 1.94 & - & - & - & - & - & - & - & - & - & - \\
\hline 18 & Poirazi [34, 35] & - & 7 & - & $\begin{array}{l}\underset{N}{N} \\
\substack{\infty \\
\infty}\end{array}$ & 1.4 & 7.5 & 60 & 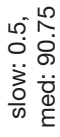 & - & 0.05 & 7 & 3 & - & - \\
\hline 19 & Jarsky [23] & - & 40 & - & - & 40 & 40 & - & - & - & - & - & - & - & - \\
\hline 20 & Royeck [14] & 1 & 100 & 0.75 & 0.05 & 5 & 5 & 2 & 0.7 & 10 & $1.1 \cdot 10^{-5}$ & $6.62 \cdot 10^{-5}$ & $4.4 \cdot 10^{-5}$ & - & $15.4 \cdot 10^{-5}$ \\
\hline 21 & Migliore_e [27] & - & 20 & - & 0.06 & 10 & 15 & 10 & - & - & - & - & - & - & - \\
\hline 22 & Miceli [17] & - & 135 & - & 0.01 & 60 & 30 & 14 & 0.01 & - & 1 & - & - & - & - \\
\hline 23 & Fineberg [18] & - & 32 & - & - & 10 & 144 & - & - & - & - & - & - & - & - \\
\hline 24 & Ashhad [30] & - & 90 & - & - & 3 & 22 & - & - & - & 1 & 0.632 & - & - & - \\
\hline 25 & Berzhanskaya [43] & - & 20 & - & 0.05 & 10 & 25 & - & - & - & - & - & - & - & - \\
\hline 26 & Marasco [38] & - & 25 & - & 0.05 & 10 & 10 & - & - & - & - & - & - & - & - \\
\hline 27 & Vladimirov_a [42] & - & 200 & - & - & 170 & 200 & 10 & 0.2 & 16 & - & 4 & - & - & - \\
\hline 28 & Vladimirov_b [45] & - & 70 & - & - & 170 & 0.5 & - & 0.8 & 20 & - & 1 & - & - & - \\
\hline 29 & Hao $[39]$ & - & 30 & - & 0.02 & 5 & 5 & - & - & - & - & - & - & - & - \\
\hline 30 & Lee - deep [44] & - & 337.5 & - & 0.038 & $90^{*}$ & 10.8 & - & 70 & 30 & - & 11.25 & - & - & - \\
\hline 31 & $\begin{array}{c}\text { Lee - superficial } \\
{[44]}\end{array}$ & - & 264 & - & 0.05 & $30^{\star}$ & 6 & - & 70 & 1 & - & 7.5 & - & - & - \\
\hline
\end{tabular}

${ }^{*}$ a slow delayed rectifier conductance also implemented

Table IV. Active conductances implemented in the axon of the model cells and their maximum conductance (mS/cm2)

\begin{tabular}{|c|c|c|c|c|c|c|c|}
\hline & Author & $\begin{array}{c}\text { persis- } \\
\text { tent } \mathrm{Na}+\end{array}$ & transient $\mathrm{Na}+$ & $\begin{array}{c}\mathrm{Na}+\text { with } \\
\text { intermediate } \\
\text { inactivation }\end{array}$ & $\begin{array}{c}\text { delayed rectifier } \\
\mathrm{K}+\end{array}$ & A type K+ & M type K+ \\
\hline 1 & Magee [40] & \multicolumn{6}{|c|}{ no axon } \\
\hline 2 & London [41] & \multicolumn{6}{|c|}{ no axon } \\
\hline 3 & Migliore_a [19] & & 64 & & 10 & 96 & - \\
\hline 4 & Watanabe [31] & - & 64 & - & 10 & 96 & - \\
\hline 5 & Poolos [28] & - & 64 & - & 3 & 16 & - \\
\hline 6 & Migliore_b [25] & & 64 & & 10 & 96 & - \\
\hline 7 & Migliore_c [26] & & 60 & & 1 & 2 & - \\
\hline 8 & Gasparini [29] & - & 60 & - & 5 & 44 & - \\
\hline 9 & Migliore_d [27] & - & 50 & - & 10 & 30 & - \\
\hline 10 & Shah_a [15] & - & 135 & - & 60 & 40 & 20 \\
\hline 11 & Ferrante [16] & - & 60 & - & 1 & 2 & - \\
\hline 12 & Marcelin [36] & \multicolumn{6}{|c|}{ passive } \\
\hline 13 & Ascoli [24] & - & 120 & - & 40 & 20 & - \\
\hline 14 & $\mathrm{Li}[32,33]$ & & 40 & & 10 & 5 & - \\
\hline 15 & Graham [37] & - & - & - & - & - & - \\
\hline 16 & Golding_a [20] & - & $\begin{array}{c}\text { hillock, AIS, internodal: } 42 \text {, } \\
\text { node:50000 }\end{array}$ & - & 40 & $\begin{array}{l}\text { hillock, AIS: } 100, \text { inter- } \\
\text { nodal, node: } 20\end{array}$ & - \\
\hline 17 & Golding_b [22] & - & - & - & - & - & - \\
\hline 18 & Poirazi $[34,35]$ & - & 100 & - & 20 & 30 & - \\
\hline 19 & Jarsky [23] & - & $\begin{array}{c}\text { hillock, AIS, intenodal:40, } \\
\text { nod:30000 }\end{array}$ & - & 40 & $\begin{array}{l}\text { hillock, AIS: 48, inter- } \\
\text { nodal, nod:9.6 }\end{array}$ & - \\
\hline 20 & Royeck [14] & AIS: 0.5 & AIS: $20-10000^{\star}$, axon: 80 & AIS: 0.5 & AIS: 20 , axon: 25 & AIS, axon: 20 & AIS, axon: 4 \\
\hline 21 & Migliore_e [27] & - & 100 & - & 50 & 75 & 10 \\
\hline 22 & Miceli [17] & - & 135 & - & 60 & 30 & 42 \\
\hline 23 & Fineberg [18] & - & 64 & - & 10 & 28.8 & - \\
\hline 24 & Ashhad [30] & - & 450 & - & 3 & - & - \\
\hline 25 & Berzhanskaya [43] & \multicolumn{6}{|c|}{ no axon } \\
\hline 26 & Marasco [38] & - & 50 & - & 10 & 30 & - \\
\hline 27 & Vladimirov_a [42] & - & 300 & - & 450 & 0.6 & 40 \\
\hline 28 & Vladimirov_b [45] & - & 300 & - & 400 & - & - \\
\hline 29 & $\mathrm{Hao}[39]$ & - & 6 & - & 5 & 5 & - \\
\hline 30 & Lee - deep [44] & - & - & - & - & - & - \\
\hline 31 & Lee - superficial [44] & - & - & - & - & - & - \\
\hline
\end{tabular}


Table V. Active conductances implemented in the dendrites of the model cells and their maximum conductance $\left(\mathrm{mS} / \mathrm{cm}^{2}\right)$

\begin{tabular}{|c|c|c|c|c|c|c|c|c|c|c|c|c|c|}
\hline No & Author & 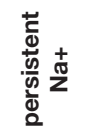 & 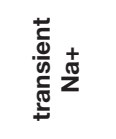 & ع & 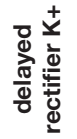 & 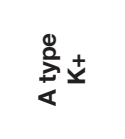 & $\underset{\Sigma}{\stackrel{0}{\geq}+}$ & $\begin{array}{l}\frac{0}{9} \\
\text { 交 } \\
\dot{ \pm}\end{array}$ & 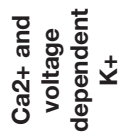 & 产 ญ & 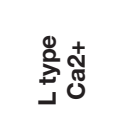 & 邑 స్ & z \\
\hline 1 & Magee [40] & - & - & - & - & - & - & - & - & - & - & - & - \\
\hline 2 & London [41] & - & - & - & - & - & - & - & - & - & - & - & - \\
\hline 3 & $\begin{array}{c}\text { Migliore_a } \\
\text { [19] }\end{array}$ & - & 32 & - & 10 & $48^{\star}+$ & - & - & - & - & - & - & - \\
\hline 4 & $\begin{array}{c}\text { Watanabe } \\
{[31]}\end{array}$ & - & 32 & - & 10 & $48^{\star}+$ & - & - & - & - & - & - & - \\
\hline 5 & Poolos [28] & - & 32 & $0.6 \#$ & $3 \#$ & $8+$ & - & - & - & - & - & - & - \\
\hline 6 & $\begin{array}{c}\text { Migliore_b } \\
\text { [25] }\end{array}$ & - & 32 & $0.5 \Delta$ & 10 & $48^{\star}+$ & - & - & - & - & - & - & - \\
\hline 7 & $\begin{array}{c}\text { Migliore_c } \\
{[26]}\end{array}$ & - & 32 & $0.05 \Delta$ & 1 & 10 & - & - & - & - & - & - & - \\
\hline 8 & Gasparini [29] & - & $30 \Delta$ & $0.05 \Delta$ & 5 & $22^{*}+$ & - & - & - & - & - & - & - \\
\hline 9 & $\begin{array}{c}\text { Migliore_d } \\
{[21]}\end{array}$ & - & 25 & $0.05 \Delta$ & 10 & $30^{\star}+$ & - & - & - & - & - & - & - \\
\hline 10 & Shah_a [15] & - & $\begin{array}{l}\text { a: } 45, \\
b: 0\end{array}$ & a: $0.05 \Delta$ & a: 60 & a: $40^{\star}+$ & - & 0.01 & - & 0.1 & - & & \\
\hline 11 & Ferrante [16] & - & 30 & $0.06 \Delta$ & $\mathrm{b}: 1$ & $\begin{array}{c}a: \\
10^{\star}+b: 10\end{array}$ & - & - & - & - & - & - & - \\
\hline 12 & Marcelin [36] & - & - & $0.05 \&$ & - & - & - & - & - & - & - & - & - \\
\hline 13 & Ascoli [24] & - & 24 & $0.02 \ddagger$ & 8 & $\begin{array}{c}\text { a: } 10^{\star}+\mathrm{b}: \\
10\end{array}$ & - & - & - & - & - & - & - \\
\hline 14 & $\mathrm{Li}[32,33]$ & - & 20 & $0.05 \Delta$ & 10 & $10^{*}+$ & - & - & - & - & - & - & - \\
\hline 15 & Graham [37] & $5 \cdot 10-3$ & - & $0.03+$ & - & $0.2+$ & - & - & - & 1 & - & - & \\
\hline 16 & $\begin{array}{c}\text { Golding_a } \\
{[20]}\end{array}$ & - & 42 & - & 40 & $100^{\star}+$ & - & - & - & - & - & - & - \\
\hline 17 & $\begin{array}{c}\text { Golding_b } \\
\text { [22] }\end{array}$ & - & - & $1.94 \ddagger$ & - & - & - & - & - & - & - & - & - \\
\hline 18 & $\begin{array}{l}\text { Poirazi [34, } \\
\text { 35] }\end{array}$ & 0.4 & 7 & $18.72 \ddagger$ & 0.86 & $7.5^{\star}+$ & $\begin{array}{c}\mathrm{a}: \\
60, \\
\mathrm{o}: \\
120\end{array}$ & $\begin{array}{c}\text { s: } 0.5- \\
0.05 \\
\text { m: 33- } \\
4.125\end{array}$ & & $0.1 \#$ & $\begin{array}{l}0.032- \\
1.452\end{array}$ & a: 0.3 & \\
\hline 19 & Jarsky [23] & - & $\begin{array}{c}\text { b: } 40, a: \\
40+\end{array}$ & - & $\mathrm{b}: 40$ & $\begin{array}{c}\text { b: } 40, \text { a: } \\
40^{*}+\end{array}$ & - & - & - & - & - & - & - \\
\hline 20 & Royeck [14] & - & 5.2085 & $1.1 \cdot 10^{-4}$ & 0.5 & $40-60$ & - & - & - & $1.1 \cdot 10^{-4}$ & $6.62 \cdot 10^{-5}$ & $4.4 \cdot 10^{-5}$ & - \\
\hline 21 & $\begin{array}{l}\text { Migliore_e } \\
{[27]}\end{array}$ & - & 20 & $\begin{array}{l}\text { b: } 0.06 \\
\text { a: } 0.06 \infty\end{array}$ & 10 & $30^{*} \&$ & - & - & - & - & - & - & 2 \\
\hline 22 & Miceli [17] & - & $\begin{array}{c}\text { b: } 0, \text { a: } \\
45\end{array}$ & $\begin{array}{l}\text { b:0, a: } \\
0.01 \Delta\end{array}$ & $\begin{array}{l}\mathrm{b}: 0 \\
\mathrm{a}: 20\end{array}$ & a: $30^{\star} \Delta$ & - & 0.01 & - & 0.1 & - & - & - \\
\hline 23 & Fineberg [18] & - & 32 & - & 10 & $\begin{array}{c}\text { b: } 144, \text { a: } \\
144+\end{array}$ & - & - & - & - & - & - & - \\
\hline 24 & Ashhad [30] & - & a: 90 & - & - & $\begin{array}{l}\mathrm{b}: 22, \mathrm{a}: \\
22^{\star} \&, 0 \ll\end{array}$ & - & - & - & $\begin{array}{c}a: 0.1+ \\
\text { b: } 0.1\end{array}$ & $\begin{array}{c}\text { a: } 0.316+ \\
\text { b: } 0.732\end{array}$ & - & - \\
\hline 25 & $\begin{array}{l}\text { Berzhans- } \\
\text { kaya [43] }\end{array}$ & - & 20 & $0.05 \Delta$ & 10 & $25^{\star}+$ & - & - & - & - & - & - & - \\
\hline 26 & Marasco [38] & - & 25 & $0.05 \Delta$ & 10 & $\begin{array}{c}\text { b: } 30, a: \\
30^{\star}+\end{array}$ & - & - & - & - & - & - & - \\
\hline 27 & $\begin{array}{c}\text { Vladimirov_a } \\
{[42]}\end{array}$ & - & 30 & - & 75 & 8 & 20 & 0.2 & 16 & - & 4 & - & - \\
\hline 28 & $\begin{array}{c}\text { Vladimirov_b } \\
{[45]}\end{array}$ & - & $\begin{array}{c}\text { b: } 10, a: \\
30\end{array}$ & - & 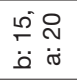 & 0.5 & - & 0.8 & $12-8-4$ & - & 1 & & \\
\hline 29 & Hao [39] & - & 30 & $0.02 \infty$ & 5 & $5 * \&$ & - & - & - & - & - & - & - \\
\hline 30 & $\begin{array}{c}\text { Lee - deep } \\
{[44]}\end{array}$ & - & - & $0.38 \#$ & $90 \S$ & $10.8^{*} \$$ & - & 70 & 30 & $50 @$ & 11.25 & - & - \\
\hline 31 & $\begin{array}{l}\text { Lee - superfi- } \\
\quad \text { cial }[44]\end{array}$ & - & - & $0.05 \#$ & $30 \S$ & $6^{\star} \$$ & - & 70 & 10 & 50@ & 7.5 & & \\
\hline
\end{tabular}

$\mathrm{a}$ - apical dendritic tree, $\mathrm{o}-$ oblique dendrites, $\mathrm{b}$ - basal dendritic tree

+ linearly increasing with the distance of the compartment from the cell body (d) with $d / 100$

$\$$ idem with $d / 200$

\# idem with $1,5 \mathrm{~d} / 100$

$\triangle$ idem with $3 \mathrm{~d} / 100$

\& idem with $5 d / 100$

$\ddagger$ increases exponentially in the apical trunk

" 3 times as at branching point

$\infty$ increases exponentially with distance from soma

@ decreases exponentially with distance from soma

* the A type $\mathrm{K}^{+}$conductance is described with different equations for the proximal (distance from soma less then 100 $\left.\mu \mathrm{m}\right)$ and distal dendritic tree

$\S$ also contains a slow component for KDR current, with the same conductance

For AHP potassium current: $\mathrm{s}$ - for the current responsible for slow AHP, $m$ - for the current responsible for medium AHP

If several values are indicated, the change is linear along the dendritic tree (proximal $\rightarrow$ distal) 
the pathomechanism of Alzheihmer's disease [48, 49], to calculate the magnetic field produced by the neuronal currents, and to estimate the phase and magnitude of the MR signals [50], to investigate the possible cognitive effects of low frequency external electric fields, such as those generated by power lines [51], to map realistic neurons into equivalent reduced models [38].

Poirazi $[34,35]$ developed a very complex, morphologically detailed model of a CA1 pyramidal cell with 17 types of ion channels, most of them distributed non-uniformly along the somato-dendritic axis. The authors originally studied the linear and non-linear synaptic integration, but being a very detailed model, it was suitable to study dendritic integration by several other authors, e.g. the thin dendritic branches as a separate integration level of the synaptic inputs, with sigmoidal summation of the neighboring inputs [52], the influence of synchronization and different spatial distribution of synaptic inputs on the proximal and distal dendrites on the information content of the neuronal response [53], complex (non-linear) as opposed to a passive (linear) information processing [54], the distance-dependent synaptic scaling [55], depolarization block [56]. This model was also used to study the alterations of the CA1 pyramidal cells in different pathological conditions, e.g. the amyloid- $\beta$-induced enhancement of release probability of CA3-CA1 synapses, and alteration of synaptic integration for inputs in the theta and gamma frequency range [57], aging-induced alterations in calciumdependent membrane mechanisms [58].

Shah_a [15] model includes similar sodium, delayed rectifier and A-type conductances as the models developed by Migliore, but a h-current with different kinetics, and also a low-threshold $\mathrm{Ca}^{2+}$ conductance, a Ca-dependent $\mathrm{K}^{+}$ conductance, and a simple $\mathrm{Ca}^{2+}$ extrusion mechanism is included. Initially the role of the axonal M-type potassium current in determining the firing properties of the neuron was explored, but later the effect of the same current on the integration of EPSPs was also studied [59]. This model was also used to determine the ion channel properties and kinetics needed to reproduce the experimentally observed depolarization block [56].

Royeck [14] developed a morphologically realistic model that contains a large number of conductances with complex $\mathrm{Ca}^{2+}$-dynamics, most of them being described by different equations from the previous models. The authors studied the relation between the voltage dependence of the sodium current and the properties of the action potential, respectively the repetitive discharge properties of CA1 neu-

Table VI. The properties of the action potentials generated by different models.

\begin{tabular}{lcccc}
\hline & Migliore & Poirazi & Shah & Royeck \\
\hline Threshold $(\mathrm{mV})$ & -33 & -43 & -43 & -44 \\
Amplitude $(\mathrm{mV})$ & 92 & 88 & 91 & 117 \\
Rate of depolarization $(\mathrm{V} / \mathrm{s})$ & 199.69 & 172.80 & 105.16 & 332.29 \\
Rate of repolarization $(\mathrm{V} / \mathrm{s})$ & -40.27 & -33.66 & -57.94 & -56.96 \\
Duration $(\mathrm{ms})$ & 1.1 & 2 & 1.1 & 1.15 \\
\hline
\end{tabular}

rons. The same model was used to investigate the effect of a mutation of the $\beta$ subunit of the sodium channel that causes heat-sensitive increase of the neuronal excitability [60], and the effect of carbamazepine on fast transient sodium currents, which mediate the fast upstroke of action potentials; and low-voltage-activated persistent sodium currents that contribute to subthreshold excitation [61].

\section{Electrophysiological properties}

Because there is a complex interaction between the passive and active membrane properties and the geometry of the model cell, the electrophysiological properties cannot be predicted even from the detailed parameter set of the model, thus these should be tested directly.

We studied in detail the above presented, most frequently used models. These differed in the passive and active properties and the geometry of the dendritic tree, but also had some similar features. All simulated models included an active axon with detailed geometry, but the dendritic spines were not considered in any of these models. The parameters which describe the passive properties of the models had constant values in three models whereas in the Poirazi model there was a sigmoidal variation of the axial resistance and the specific membrane resistance. (Table VI)

Most of the active conductances implemented in the simulated models were described by different equations, except the A-type $\mathrm{K}^{+}$-conductance. Poirazi and Royeck implemented a detailed model of $\mathrm{Ca}^{2+}$ dynamics, the Shah model contains only a T-type $\mathrm{Ca}^{2+}$-conductance, whereas in the Migliore model only $\mathrm{Na}^{+}$, delayed rectifier, A-type $\mathrm{K}^{+}$and $\mathrm{h}$-conductances are inserted.

Despite the large variation of the active conductances implemented in the models, the properties of the action potentials were quite similar: there were no important differences in the action potential threshold and amplitude. Nevertheless, the rate of depolarization was much slower in the Shah model, and the duration of the action potential was longer for the Poirazi model. (Table VII, Figure 1)

The tested models had different firing properties and none of them could reproduce all of the studied phenomena. The maximum firing rate of the models was close to the experimentally described values, except for the Shah model, which had significantly lower firing rate. The train of the action potentials obtained by maximum stimulus intensity showed frequency adaptation only in case of the Shah and Royeck models. The decrease of the firing rate during a long current impulse is considered to be the effect of the slow activation of the $\mathrm{M}$-type and $\mathrm{Ca}^{2+}$-dependent

Table VII. The firing pattern and the back-propagation of the action potentials in the apical dendrites of the modeled neurons

\begin{tabular}{ccccc}
\hline & Migliore b & Poirazi & Shah & Royeck \\
\hline Maximum firing frequency & 111 & 127 & 35 & 127 \\
Frequency adaptation & no & no & yes & yes \\
$\begin{array}{c}\text { Progressive decrease in } \\
\text { amplitude of the back-prop- }\end{array}$ & yes & yes & no & no \\
agated action potentials & & & & \\
\hline
\end{tabular}




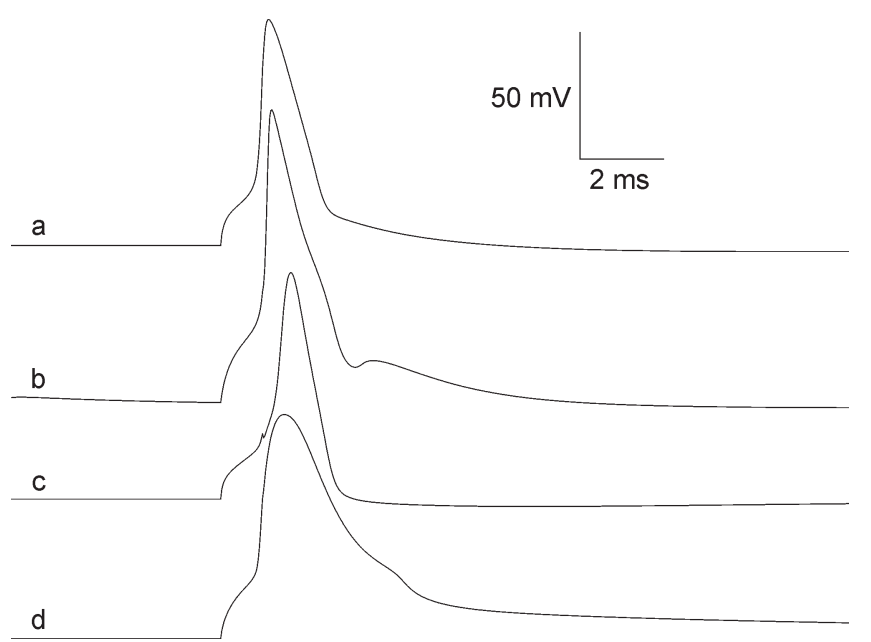

Fig. 1. The action potentials of different models. a: Shah, b: Royeck, c: Migliore_d, d: Poirazi

(AHP) $\mathrm{K}^{+}$-conductances. These conductances were implemented also in the Poirazi model, but the model's firing did not adapt during a long train. As expected, the Migliore model, in which these conductances were not implemented, did not show the adaptation phenomenon.

Regarding the back-propagation of the action potentials, a pronounced decrease of action potential amplitude

A

d

c
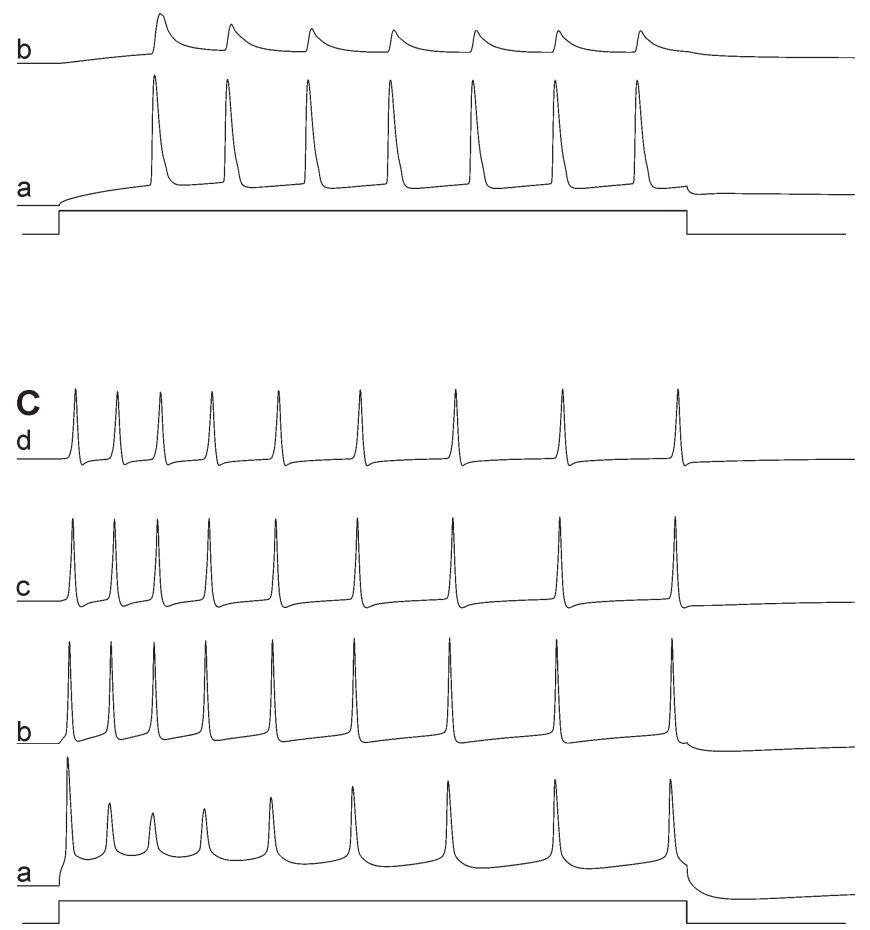

along the dendritic tree was found in all models except the Shah model. This non-linear decrease was caused by the non-uniform distribution of the dendritic A-type $\mathrm{K}^{+}$ conductances. This conductance was described by the same equation in all models, with linear increase along the apical dendritic tree, although the value of the maximum conductance differed. The non-uniform passive membrane properties of the dendrites in the Poirazi model could also contribute to this effect. (Figure 2)

In CA1 pyramidal neurons the back-propagation of the action potentials in the dendritic tree is an active process. During repetitive firing the amplitude of the action potentials decreases because of the slow inactivation of the $\mathrm{Na}^{+}$-channels. The decrease of the amplitude of the backpropagating action potentials was observed only in the Poirazi model, although the dendritic sodium conductance showed slow inactivation in all models.

\section{Discussion}

The models used to investigate the back-propagation of the action potentials in the dendritic tree are among the simplest, containing only a few active conductances. All models contain sodium, delayed rectifier and A-type potassium conductances implemented in all compartments. The

B

d

C
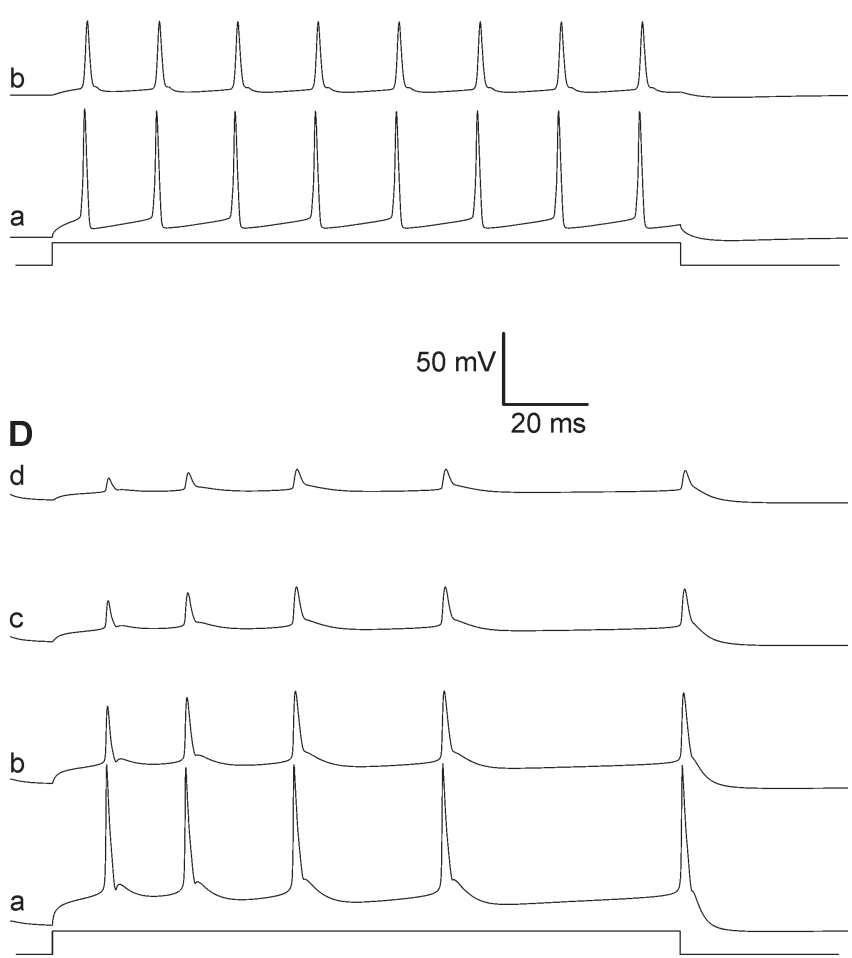

Fig. 2. The firing properties and the back-propagation of the action potentials in the apical dendrites of the modeled neurons. Simulated membrane potential recorded at: A: Poirazi, a: soma, b: $140 \mu \mathrm{m}, \mathrm{c}: 250 \mu \mathrm{m}, \mathrm{d}: 310 \mu \mathrm{m}$ from the soma in stratum radiatum; B: Migliore_d, a: soma, b: $100 \mu \mathrm{m}, \mathrm{c}: 280 \mu \mathrm{m}, \mathrm{d}: 460 \mu \mathrm{m}$ from the soma; C: Shah, a: soma, b: $155 \mu \mathrm{m}, \mathrm{c}: 300 \mu \mathrm{m}, \mathrm{d}: 430 \mu \mathrm{m}$ from the soma; D: Royeck, a: soma, b: $115 \mu \mathrm{m}, \mathrm{c}: 190 \mu \mathrm{m}, \mathrm{d}$ : $250 \mu \mathrm{m}$ from the soma. Bottom trace on each panel indicates the duration of the current injection. All figures are on the same scale. 
$\mathrm{Ca}^{2+}$-dynamics is not simulated in any of the models. The A-type potassium conductance implemented in the models has similar distribution (linear increase with distance from soma along the apical dendritic tree) and kinetics (different for the proximal and distal dendrites, the current in the distal dendrites activates at a more negative membrane potential). In the Migliore_d model a h-type conductance is also implemented with a linear increase along the dendritic tree with distance from soma. The Migliore_d model was also used to investigate dendritic integration in physiological and pathological conditions. Because of its relative simplicity, this model, which was validated in a large number of different studies, is especially suitable to study the interactions between the neuronal activity and the external electrical or magnetic field.

The models focusing on signal generation (firing properties) are more complex. Most of these models contain besides the sodium, delayed rectifier and A-type potassium conductances also $\mathrm{M}$-type $\mathrm{K}^{+}$, AHP $\mathrm{K}^{+}$and at least one $\mathrm{Ca}^{2+}$ conductance to simulate the effect of $\mathrm{Ca}^{2+}$-dependant $\mathrm{K}^{+}$-conductances on the firing rate. The models that do not contain $\mathrm{Ca}^{2+}$-dependant conductances investigate the effect of several drugs modulating the synaptic transmission or the effect of ion channels described by different kinetics on the firing properties. We tested two of these models and they both show the experimentally described spike frequency adaptation, but the progressive decrease of back-propagating action potentials and a high frequency (around $100 \mathrm{~Hz}$ ) action potential train is shown only by the Royeck model.

Most models are developed to study the somato-dendritic signal integration. Two of these studies investigate the synaptic scaling of excitatory synaptic inputs in a passive model, in two other models only an h-current is implemented. The dynamics of the $\mathrm{Ca}^{2+}$-ions is not simulated in most of the remaining models. Only two models contain several $\mathrm{Ca}^{2+}$-conductances with non-uniform distribution in the dendritic tree. One of these models (Ashhad) was developed to study the $\mathrm{Ca}^{2+}$-spikes, the other is the Poirazi model, the most complex of the studied models. This model was developed to study the dendritic integration of excitatory synaptic inputs. The Poirazi model was used in several studies all focusing on untangling the mechanisms of dendritic integration. Although this model contains the most diverse conductances, during maximum current injection does not present the spike frequency adaptation.

Our results show that simpler models still exhibit many of the important features of CA1 pyramidal neurons, thus these models can be used to simulate the pathomechanism of different diseases that alter neuronal excitability; and because of the relatively low computational costs they can be also used for modeling clusters of similarly functioning cells. These models can also be used in studies that focus on a specific modification - a drug effect or a mutation affecting the kinetics of an ion channel.
If the initiation and propagation of dendritic spikes is investigated, the model should reproduce the dynamics of $\mathrm{Ca}^{2+}$-ions in the dendrites in a complex manner, like the models developed by Ashhad or Poirazi. The Poirazi model is best suited to simulate the information processing in thin dendritic branches or the synaptic integration of a large number of synaptic inputs with varying spatiotemporal distribution.

\section{Conclusion}

Each studied model was optimized to explore particular physiological and/or pathological properties of the CA1 pyramidal cells. Not even the most complex models could reproduce all studied biological phenomena, therefore we conclude, that at the moment the "perfect" pyramidal cell model is not yet available. Further validation against biological datasets is necessary, and the models still have to be tuned to eliminate the identified inconsistencies.

As a general purpose model cell does not exist yet, the researchers have to choose which model is the most suitable for the scientific question under investigation. Our work, hopefully, will help finding the best model for each in silico experiment.

\section{References}

1. D'Angelo E, Solinas S, Garrido J et al. Realistic modeling of neurons and networks: towards brain simulation. Funct Neurol. 2013; 28(3):153-166.

2. Gewaltig MO, Cannon R. Current practice in software development for computational neuroscience and how to improve it. PLoS Comput Biol. 2014; 10(1):e1003376.

3. Gleeson P, Crook S, Cannon RC et al. NeuroML: a language for describing data driven models of neurons and networks with a high degree of biological detail. PLoS Comput Biol. 2010; 6(6):e1000815.

4. O'Keefe J, Dostrovsky J. The hippocampus as a spatial map. Preliminary evidence from unit activity in the freely-moving rat. Brain Res. 1971; 34(1):171-175.

5. Scoville WB, Milner B. Loss of recent memory after bilateral hippocampal lesions. J Neurol Neurosurg Psychiatry. 1957; 20(1):11-21.

6. Corkin S. What's new with the amnesic patient H.M.? Nat Rev Neurosci. 2002; 3(2):153-160.

7. Green JD. The Hippocampus. Physiol Rev. 1964; 44:561-608.

8. Tellez-Zenteno JF, Hernandez-Ronquillo L. A review of the epidemiology of temporal lobe epilepsy. Epilepsy Res Treat. 2012; doi:10.1155/2012/630853.

9. Braak H, Braak E. Neuropathological stageing of Alzheimer-related changes. Acta Neuropathol. 1991; 82(4):239-259.

10. Hardy J, Selkoe DJ. The amyloid hypothesis of Alzheimer's disease: progress and problems on the road to therapeutics. Science. 2002; 297(5580):353-356.

11. Harrison PJ, Law AJ, Eastwood SL. Glutamate receptors and transporters in the hippocampus in schizophrenia. Ann N Y Acad Sci. 2003; 1003:94101.

12. Carnevale NT, Hines ML. The NEURON Book: Cambridge University Press; 2006.

13. What is NEURON? 2014 [accessed on 2014.11.10]; Available from: http://www.neuron.yale.edu/neuron/what_is_neuron.

14. Royeck M, Horstmann MT, Remy S et al. Role of axonal NaV1.6 sodium channels in action potential initiation of CA1 pyramidal neurons. J Neurophysiol. 2008; 100(4):2361-2380.

15. Shah MM, Migliore M, Valencia I et al. Functional significance of axonal Kv7 channels in hippocampal pyramidal neurons. Proc Natl Acad Sci U S A. 2008; 105(22):7869-7874.

16. Ferrante M, Blackwell KT, Migliore M, Ascoli GA. Computational models of neuronal biophysics and the characterization of potential neuropharmacological targets. Curr Med Chem. 2008; 15(24):2456-2471.

17. Miceli F, Soldovieri MV, Lugli L et al. Neutralization of a unique, negatively- 
charged residue in the voltage sensor of $\mathrm{K} \mathrm{V} 7.2$ subunits in a sporadic case of benign familial neonatal seizures. Neurobiol Dis. 2009; 34(3):501510.

18. Fineberg JD, Ritter DM, Covarrubias M. Modeling-independent elucidation of inactivation pathways in recombinant and native A-type Kv channels. J Gen Physiol. 2012; 140(5):513-527.

19. Migliore M, Hoffman DA, Magee JC, Johnston D. Role of an A-type K+ conductance in the back-propagation of action potentials in the dendrites of hippocampal pyramidal neurons. J Comput Neurosci. 1999; 7(1):5-15.

20. Golding NL, Kath WL, Spruston N. Dichotomy of action-potential backpropagation in CA1 pyramidal neuron dendrites. J Neurophysiol. 2001; 86(6):2998-3010.

21. Migliore M, Ferrante M, Ascoli GA. Signal propagation in oblique dendrites of CA1 pyramidal cells. J Neurophysiol. 2005; 94(6):4145-4155.

22. Golding NL, Mickus TJ, Katz Y et al. Factors mediating powerful voltage attenuation along CA1 pyramidal neuron dendrites. J Physiol. 2005; 568(Pt 1):69-82

23. Jarsky T, Roxin A, Kath WL, Spruston N. Conditional dendritic spike propagation following distal synaptic activation of hippocampal CA1 pyramidal neurons. Nat Neurosci. 2005; 8(12):1667-1676.

24. Ascoli GA, Gasparini S, Medinilla V, Migliore M. Local control of postinhibitory rebound spiking in CA1 pyramidal neuron dendrites. J Neurosci. 2010; 30(18):6434-6442.

25. Migliore M. On the integration of subthreshold inputs from Perforant Path and Schaffer Collaterals in hippocampal CA1 pyramidal neurons. J Comput Neurosci. 2003; 14(2):185-192.

26. Migliore M, Messineo L, Ferrante M. Dendritic In selectively blocks temporal summation of unsynchronized distal inputs in CA1 pyramidal neurons. J Comput Neurosci. 2004; 16(1):5-13.

27. Migliore M, Migliore R. Know your current I(h): interaction with a shunting current explains the puzzling effects of its pharmacological or pathological modulations. PLoS One. 2012; 7(5):e36867.

28. Poolos NP, Migliore M, Johnston D. Pharmacological upregulation of h-channels reduces the excitability of pyramidal neuron dendrites. Nat Neurosci. 2002; 5(8):767-774.

29. Gasparini S, Migliore M, Magee JC. On the initiation and propagation of dendritic spikes in CA1 pyramidal neurons. J Neurosci. 2004; 24(49):11046-11056.

30. Ashhad S, Narayanan R. Quantitative interactions between the A-type K+ current and inositol trisphosphate receptors regulate intraneuronal $\mathrm{Ca}+$ waves and synaptic plasticity. J Physiol. 2012; 591(Pt 7):1645-1669.

31. Watanabe S, Hoffman DA, Migliore M, Johnston D. Dendritic $\mathrm{K}+$ channels contribute to spike-timing dependent long-term potentiation in hippocampal pyramidal neurons. Proc Natl Acad Sci U S A. 2002; 99(12):8366-8371.

32. Li X, Ascoli GA. Computational simulation of the input-output relationship in hippocampal pyramidal cells. J Comput Neurosci. 2006; 21(2):191-209.

33. Li X, Ascoli GA. Effects of synaptic synchrony on the neuronal input-output relationship. Neural Comput. 2008; 20(7):1717-1731.

34. Poirazi P, Brannon T, Mel BW. Pyramidal neuron as two-layer neural network. Neuron. 2003; 37(6):989-999.

35. Poirazi P, Brannon T, Mel BW. Arithmetic of subthreshold synaptic summation in a model CA1 pyramidal cell. Neuron. 2003; 37(6):977-987.

36. Marcelin B, Chauviere L, Becker A et al. h channel-dependent deficit of theta oscillation resonance and phase shift in temporal lobe epilepsy. Neurobiol Dis. 2009; 33(3):436-447.

37. Graham BP. Pattern recognition in a compartmental model of a CA1 pyramidal neuron. Network. 2001; 12(4):473-492.

38. Marasco A, Limongiello A, Migliore M. Using Strahler's analysis to reduce up to 200-fold the run time of realistic neuron models. Sci Rep. 2013; 3:2934.

39. Hao J, Wang XD, Dan $Y$ et al. An arithmetic rule for spatial summation of excitatory and inhibitory inputs in pyramidal neurons. Proc Natl Acad Sci U S A. 2009; 106(51):21906-21911.

40. Magee JC, Cook EP. Somatic EPSP amplitude is independent of synapse location in hippocampal pyramidal neurons. Nat Neurosci. 2000; 3(9):895-
903.

41. London M, Segev I. Synaptic scaling in vitro and in vivo. Nat Neurosci. 2001; 4(9):853-855.

42. Vladimirov N, Tu Y, Traub RD. Shortest Loops are Pacemakers in Random Networks of Electrically Coupled Axons. Front Comput Neurosci. 2012; 6:17.

43. Berzhanskaya J, Chernyy N, Gluckman BJ et al. Modulation of hippocampal rhythms by subthreshold electric fields and network topology. J Comput Neurosci. 2012; 34(3):369-389.

44. Lee SH, Marchionni I, Bezaire M et al. Parvalbumin-positive basket cells differentiate among hippocampal pyramidal cells. Neuron. 2014; 82(5):1129-1144.

45. Vladimirov N, Tu Y, Traub RD. Synaptic gating at axonal branches, and sharp-wave ripples with replay: a simulation study. Eur J Neurosci. 2013; 38(10):3435-3447.

46. Migliore M, Novara G, Tegolo D. Single neuron binding properties and the magical number 7. Hippocampus. 2008; 18(11):1122-1130.

47. Migliore M, De Blasi I, Tegolo D, Migliore R. A modeling study suggesting how a reduction in the context-dependent input on CA1 pyramidal neurons could generate schizophrenic behavior. Neural Netw. 2011; 24(6):552-559.

48. Culmone V, Migliore M. Progressive effect of beta amyloid peptides accumulation on CA1 pyramidal neurons: a model study suggesting possible treatments. Front Comput Neurosci. 2012; 6:52.

49. Morse TM, Carnevale NT, Mutalik PG et al. Abnormal Excitability of Oblique Dendrites Implicated in Early Alzheimer's: A Computational Study. Front Neural Circuits. 2010; 4.

50. Cassara AM, Maraviglia B. Microscopic investigation of the resonant mechanism for the implementation of nc-MRI at ultra-low field MRI. Neuroimage. 2008; 41(4):1228-1241.

51. Cavarretta F, Carnevale NT, Tegolo D, Migliore M. Effects of low frequency electric fields on synaptic integration in hippocampal CA1 pyramidal neurons: implications for power line emissions. Front Cell Neurosci. 2014; 8:310.

52. Polsky A, Mel BW, Schiller J. Computational subunits in thin dendrites of pyramidal cells. Nat Neurosci. 2004; 7(6):621-627.

53. Pissadaki EK, Sidiropoulou K, Reczko M, Poirazi P. Encoding of spatiotemporal input characteristics by a CA1 pyramidal neuron model. PLoS Comput Biol. 2010; 6(12):e1001038.

54. Gomez Gonzalez JF, Mel BW, Poirazi P. Distinguishing Linear vs. NonLinear Integration in CA1 Radial Oblique Dendrites: It's about Time. Front Comput Neurosci. 2011; 5:44.

55. Sterratt DC, Groen MR, Meredith RM, van Ooyen A. Spine calcium transients induced by synaptically-evoked action potentials can predict synapse location and establish synaptic democracy. PLoS Comput Biol. 2012; 8(6):e1002545.

56. Bianchi D, Marasco A, Limongiello A et al. On the mechanisms underlying the depolarization block in the spiking dynamics of CA1 pyramidal neurons. J Comput Neurosci. 2012; 33(2):207-225.

57. Romani A, Marchetti C, Bianchi D et al. Computational modeling of the effects of amyloid-beta on release probability at hippocampal synapses. Front Comput Neurosci. 2013; 7:1.

58. Markaki M, Orphanoudakis S, Poirazi P. Modelling reduced excitability in aged CA1 neurons as a calcium-dependent process. Computational Neuroscience. 2005; 65-66:305-314.

59. Shah MM, Migliore M, Brown DA. Differential effects of Kv7 (M-) channels on synaptic integration in distinct subcellular compartments of rat hippocampal pyramidal neurons. J Physiol. 2011; 589(Pt 24):6029-6038.

60. Wimmer VC, Reid CA, Mitchell S et al. Axon initial segment dysfunction in a mouse model of genetic epilepsy with febrile seizures plus. J Clin Invest. 2010; 120(8):2661-2671.

61. Uebachs M, Opitz T, Royeck M et al. Efficacy loss of the anticonvulsant carbamazepine in mice lacking sodium channel beta subunits via paradoxical effects on persistent sodium currents. J Neurosci. 2010; 30(25):8489-8501. 